BI-TP 2016/02(rev)

\title{
Universal Strangeness Production in Hadronic and Nuclear Collisions
}

\author{
P. Castorina ${ }^{\mathrm{a}, \mathrm{b}}$, S. Plumari ${ }^{\mathrm{a}, \mathrm{c}}$ and H. Satz ${ }^{\mathrm{d}}$ \\ a: Dipartimento di Fisica ed Astronomia, Universitá di Catania, Italy \\ b: INFN, Sezione di Catania, Catania, Italy \\ c: INFN-LNS, Catania, Italy \\ d: Fakultät für Physik, Universität Bielefeld, Germany
}

\begin{abstract}
We show that strangeness suppression in hadronic and nuclear collisions is fully determined by the initial energy density of the collision. The suppression factor $\gamma_{s}(s)$, with $\sqrt{s}$ denoting the collision energy, can be expressed as a universal function of the initial energy density $\epsilon_{0}(s)$, and the resulting pattern is in excellent agreement with data from $p-p, p-P b, C u-C u, A u-A u$ and $P b-P b$ data over a wide range of energies and for different centralities.
\end{abstract}

The relative hadron production rates in high energy strong interactions are in general well accounted for in terms of an ideal resonance gas at temperature $T$ and baryochemical potential $\mu$, with one significant caveat. Over a wide range of collision energies, both in $p p$ and in $A A$ collisions, the rates for the production of strange hadrons are found fall below the values predicted by an ideal resonance gas in chemical equilibrium; see e.g. [1] 3]. The resonance gas scenario can be maintained, however, by the rather ad hoc introduction of a strangeness suppression factor $\gamma_{s}(s)<1$, with $\gamma_{s}^{n}$ reducing the production rate of hadrons containing $n$ strange quarks or antiquarks [4]. With increasing collision energies $\sqrt{s}$, the strangeness suppression decreases, i.e., $\gamma_{s}(s)$ is found to approach unity. In Fig. 1, we show the behavior of $\gamma_{s}(s)$ as function of the collision energy $\sqrt{s}$ in $p p$ and heavy ion $(\mathrm{Pb}-\mathrm{Pb}$, $A u-A u, C u-C u)$ collisions [5]; we return to the details of the mentioned fits a little later on. With such a suppression factor, one finds excellent agreement for the relative hadron abundances in hadronic and nuclear collisions as well as in $e^{+} e^{-}$annihilation.

Nevertheless, the origin and functional behavior of $\gamma_{s}$ has for a long time remained enigmatic, as has the difference in behavior between elementary and nuclear collisions. A first step towards a solution was obtained by noting that in collisions producing only a small number of strange particles, strangeness conservation should be enforced not only exactly (canonical instead of grand canonical) [6], but moreover on a local level, within a strangeness correlation volume $V_{c}<V$ : the production of a single strange particle would require that of an antiparticle nearby, not somewhere in some large equivalent global volume $V$ [7]. Such a requirement is effectively a deviation from global ideal gas behavior, and it seems necessary only if there are just a few strange hadrons. Once their numbers become large enough, local compensation is automatically given, so that there 


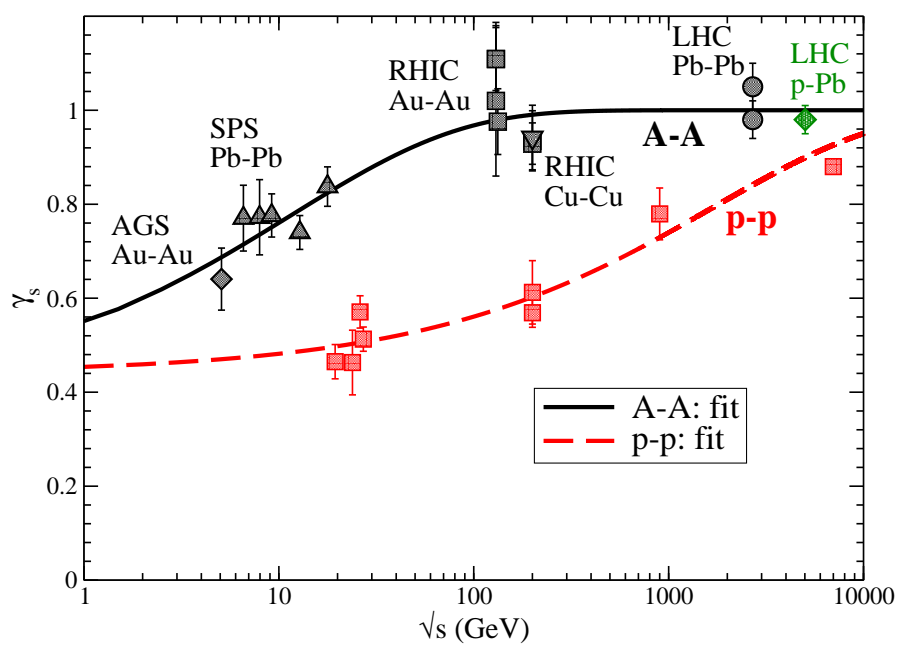

Figure 1: The strangeness suppression factor $\gamma_{s}$ as function of the collision energy $\sqrt{s}$ for $p p$ (red symbols), $\mathrm{Pb}-\mathrm{Pb}, \mathrm{Au}-\mathrm{Au}, \mathrm{Cu}-\mathrm{Cu}$ (black symbols) and $p-P b$ (green symbol) collisions [5].

is no longer any need for a specific strangeness correlation volume. And indeed one finds that for $V_{c} / V \rightarrow 1$, the corresponding resonance gas predictions converge to those of an equilibrium grand canonical formulation.

The theoretical basis for such strangeness correlation volumes was recently provided through causality considerations for the space-time evolution of high energy interactions [9, 10]. In a boost-invariant production scenario [11], one has after a brief thermalization stage an intermediate thermal medium in strong interaction; this then freezes out into free hadrons. At the thermalization time, all information about the initial state is lost, and beyond the freeze-out time, there is no further interaction between the individual hadrons. In [9, 10] it was shown that between these two times, the strongly interacting thermal medium, whether deconfined (QGP) or confined (interacting hadrons), is partioned into causally disconnected space-time regions, similar to the horizon problem in cosmology, with no communication possible between different regions. Hadrons produced at large rapidity come from a thermal fireball which is causally disjoint from a fireball leading to low rapidity hadrons, and so one cannot expect strangeness conservation to occur through interaction between the relevant bubbles. The concept of a global equivalent cluster [1] thus cannot be applied here: exactly conserved quantum numbers have to be conserved within (smaller) causally connected volumes. This implies that any dynamical correlations among regions separated by a large rapidity gap must originate before the equilibration time.

The aim of the present paper is to show that this scenario leads to a universal description of strangeness production in high energy collisions, providing a common formulation for $p p$ and $A A$ collisions at different collision energies, different centralities, and different $A$. In Fig. 2, we illustrate the definition of a fireball in terms of the collision evolution: we require a causal connection between the most separate points $\left(q_{R}\right.$ and $\left.h_{L}\right)$ of the bubble.

In such a scheme, the size $d$ of the causal correlation region is determined by the values of the thermalization time $\tau_{0}$ (the "equilibration" time) and the hadronisation time $\tau_{h}$, 


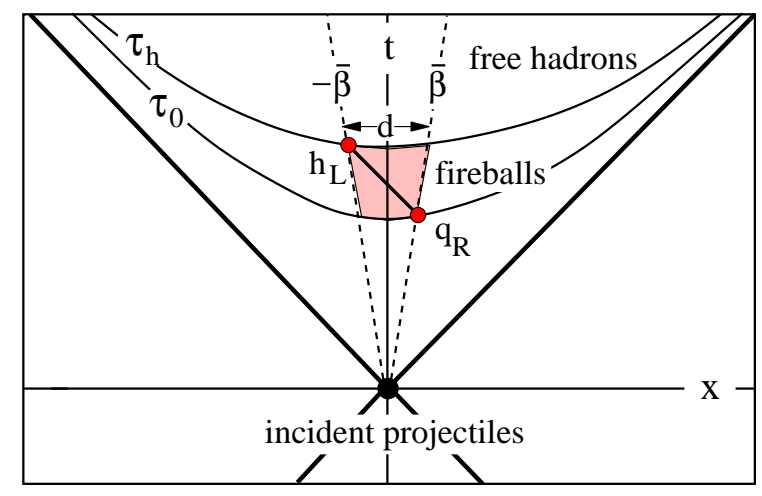

Figure 2: The formation and evolution of a fireball at rest in the center of mass; the fireball is indicated in pink.

specifying the emission of free hadrons. Geometric considerations [9] result in

$$
\frac{d}{\tau_{0}}=\sqrt{\frac{\tau_{h}}{\tau_{0}}}\left(\frac{\tau_{h}}{\tau_{0}}-1\right) .
$$

For boost-invariant hadronisation, the time evolution is governed by the one-dimensional hydrodynamic expansion

$$
\frac{d \epsilon}{d \tau}=-\frac{(\epsilon+p)}{\tau}
$$

where $\epsilon$ denotes the energy density and $p$ the pressure. Given the equation of state of the plasma, we can thus express $\tau$ as function of the energy density $\epsilon$. To illustrate, we consider an ideal quark-gluon plasma, with $p=\epsilon / 3$, to obtain

$$
\frac{\tau_{h}}{\tau_{0}}=\left(\frac{\epsilon_{0}}{\epsilon_{h}}\right)^{3 / 4}
$$

As other extreme, we set $p=0$, to find

$$
\frac{\tau_{h}}{\tau_{0}}=\left(\frac{\epsilon_{0}}{\epsilon_{h}}\right)
$$

For a realistic description, we can take the equation of state as determined in finite temperature lattice QCD studies [12,13], which with $p=a \epsilon, 0<a<1 / 3$, gives us

$$
\frac{\tau_{h}}{\tau_{0}}=\left(\frac{\epsilon_{0}}{\epsilon_{h}}\right)^{1 /(1+a)} .
$$

a value lying somewhere between the two extremes just considered. For our present considerations, however, the precise form is not important, as we shall see.

What is crucial is that the spatial size $d$ of the causally correlated region is determined by $\epsilon_{0} / \epsilon_{h}$. Since the universal hadronisation energy density $\epsilon_{h} \simeq 0.4-0.6 \mathrm{GeV} / \mathrm{fm}^{3}$ is obtained in lattice QCD studies and $\tau_{0}$ is conventionally assumed to be about $1 \mathrm{fm}$, we find that the correlation volume is effectively specified by the initial energy density $\epsilon_{0}(s)$. For a central collision in the boost-invariant scheme assumed here, this is given by the Bjorken expression [14]

$$
\epsilon_{0} \tau_{0}=\frac{1.5 A}{\pi R_{A}^{2}}\left(\frac{d E}{d y}\right)_{y=0}^{A A}
$$


with $R_{A}=1.25 A^{1 / 3}$. Here $A(d E / d y)_{y=0}^{A A}$ is the average energy deposited in the $y=0$ interval of a central $A A$ collision; hence $(d E / d y)_{y=0}^{A A}$ denotes the average energy per one-half the number of participants in the nuclear interaction volume. A simplistic approximation gives

$$
\left(\frac{d E}{d y}\right)_{y=0}^{A A} \simeq m_{T}\left(\frac{d N}{d y}\right)_{y=0}^{A A},
$$

where $m_{T} \simeq 0.5 \mathrm{GeV}$ is the average transverse energy per produced hadron and $(d N / d y)_{y=0}^{A A}$ the average multiplicity per one-half the number of participants deposited in the nuclear interaction volume. The result is the often-used form

$$
\epsilon_{0} \tau_{0}=\frac{1.5 m_{T} A}{\pi R_{A}^{2}}\left(\frac{d N}{d y}\right)_{y=0}^{A A},
$$

for the central energy density. It effectively ignores the energy density component arising from hydrodynamic flow; nevertheless, we shall use it to begin with and later on return to the modification arising when flow is included.

The average charged multiplicity in $P b-P b$ and $A u-A u$ collisions at central rapidity and per one-half the number of participants has been parametrized by [15]

$$
\left(\frac{d N}{d y}\right)_{y=0}^{A A}=a(\sqrt{s})^{0.3}+b,
$$

with $\mathrm{a}=0.7613$ and $\mathrm{b}=0.0534\left(A(d N / d y)_{y=0}^{A A}\right.$ is the overall charged multiplictiy in a central $A A$ collision). The counterpart for $p p$ collisions is

$$
\epsilon_{0}^{p} \tau_{0}=\frac{1.5 m_{T}}{\pi R_{p}^{2}}\left(\frac{d N}{d y}\right)_{y=0}^{p p}
$$

with

$$
\left(\frac{d N}{d y}\right)_{y=0}^{p p}=a(\sqrt{s})^{0.22}+b,
$$

where $R_{p}=0.8 \mathrm{fm}, \mathrm{a}=0.797$ and $\mathrm{b}=0.04123[15]$.

These expressions specify the initial energy density $\epsilon_{0}$ in terms of the collision energy $\sqrt{s}$, and this in turn gives us the causal correlation size $d$. It is thus the initial energy density which determines the degree of strangeness suppression. If this is correct, we should be able to relate directly the strangeness suppression as given by $\gamma_{s}(s)$ to the initial energy densities of $p p$ and $A A$ collisions.

To test this, we use the fits to $\gamma_{s}(s)$ given in [16, 17],

$$
\gamma_{s}^{A}(s)=1-a_{A} \exp \left(-b_{A} \sqrt{A \sqrt{s}}\right)
$$

and

$$
\gamma_{s}^{p}(s)=1-a_{p} \exp \left(-b_{p} s^{1 / 4}\right)
$$

with $a_{A}=0.606, a_{p}=0.5595, b_{A}=0.0209, b_{p}=0.0242$. In Fig. 1, these fits were shown together with high energy data. At a given collision energy $s$, we thus have from equ'ns. (6-11) the average energy density $\epsilon_{0}(s)$ and from equ'ns. (12-13) the corresponding value 


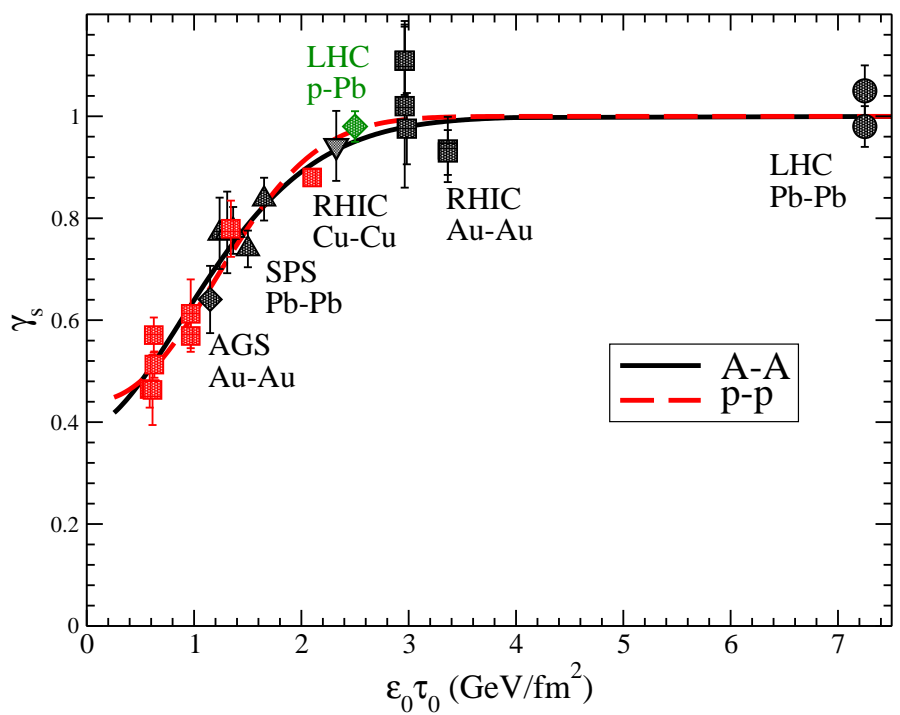

Figure 3: Strangeness suppression as function of the initial energy density in $p p, p P b$ and $A A$ collisions, based on eq.(8); black symbols correspond to $A A$, green to $p P b$ and red to pp data.

of $\gamma_{s}(s)$. As a result, we therefore obtain $\gamma_{s}(s)$ as a function of $\epsilon_{0}(s)$. The result is shown in Fig. 3 together with the available data from SPS to LHC energies.

In Fig. 1, we had also included the LHC point for $p-P b$ collisions at $\sqrt{s}=5.02 \mathrm{TeV}$. To determine the corresponding energy density, we use in eq. (6)

$$
R_{T}=R_{p}\left(0.5 \bar{N}_{\text {part }}\right)^{1 / 3}
$$

for the transverse radius, with $\bar{N}_{\text {part }} \simeq 8$, as given by [18], together with the average secondary multiplicity found there. The resulting point is included in Fig. 3 .

In Fig. 3, we note first of all that the functional forms of $\gamma_{s}$ for $A A$ and $p p$ collisions in terms of $\epsilon_{0}(s) \tau_{0}$ fully coincide; the difference between elementary and nuclear collisions seen in Fig. 1 is simply due to the fact that strangeness suppression is not determined by the overall collision energy. Instead, it is governed by the initial energy density. Next we note that in fact all data are in excellent agreement with such a universal energy density scaling of strangeness suppression.

As a further test, we can check the variation of $\gamma_{s}$ with the centrality of the collision at fixed $A$ and $\sqrt{s}$. This requires a definition of the initial energy density for non-central collisions. In the spirit of our form (마), we have used

$$
\epsilon_{0}^{N_{p}} \tau_{0}=\frac{1.5 m_{T}\left(0.5 N_{p}\right)}{\pi R_{N_{p}}^{2}}\left(\frac{d N}{d y}\right)_{y=0}^{A A},
$$

with $R_{N_{p}}=1.25\left(0.5 N_{p}\right)^{1 / 3}$ as an estimate of the energy density as function of the number of participants $N_{p}$. This then allows us to enter recent data for $\gamma_{s}$ as function of $N_{p}$ in $A u-A u$ and $C u-C u$ collisions at $200 \mathrm{GeV}$ [19]. In Fig. 4 it is seen to agree quite well with the universal curve obtained from the central data. - In the similar vein, it would be interesting to see if $p p$ data at fixed $\sqrt{s}$ also follow the predicted pattern; for fixed transverse area, $\gamma_{s}$ must increase with multiplicity according to the curve shown in Figs. 3 and 4, see eq. (10). 


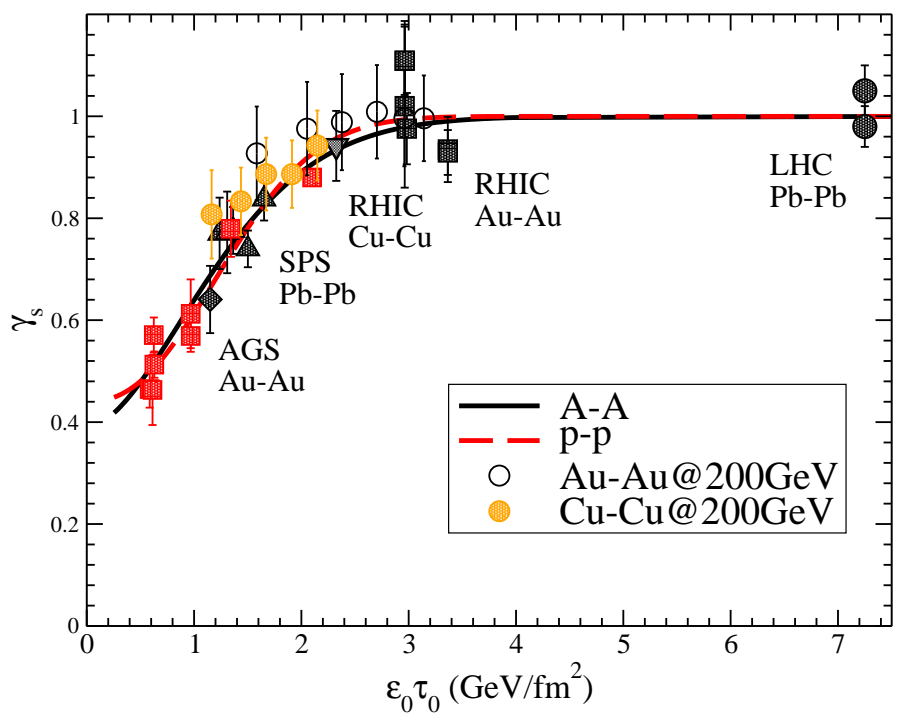

Figure 4: Strangeness suppression as function of the initial energy density in central $p p$ and $A A$ collisions, as in Fig. 3, the circles show the results from non-central collisions, using eq.(15).

We now return to the assumed form (8) for the energy density. In a hydrodynamic description, it is the entropy density which is related to the number of emitted secondaries,

$$
s_{0}=c_{s} T^{3}=\frac{c}{\tau_{0} \pi R_{A}^{2}}\left(\frac{d N}{d y}\right) ;
$$

this holds for an ideal gas of massless hadrons at temperature $T$, with $c_{s}$ and $c$ determined by the number of degrees of freedom of the species produced; the transverse nuclear area is given by $\pi R_{A}^{2}$. The corresponding energy density is then given by

$$
\epsilon_{0}=(3 / 4) c_{s} T^{4}
$$

so that the relation between energy density and multiplicity is now given by

$$
\epsilon_{0} \tau_{0}=(3 / 4) \frac{\tau_{0}}{c_{s}^{1 / 3}}\left[\frac{c}{\tau_{0} \pi R_{A}^{2}}\left(\frac{d N}{d y}\right)\right]^{4 / 3} .
$$

Comparing equ's. (8) and (18) we find that as before, when $(d N / d y)(s)$ is given, $\gamma_{s}(s)$ and $\epsilon_{0}(s)$ are fully specified for a given $s$. Our main result, strangeness suppression is determined by the initial energy density, thus remains valid. More generally speaking, $\gamma_{s}(s)$ at given $s$ is uniquely correlated to the initial energy density at this $s$, as defined in eq. (6) , i.e., to the total (transverse) energy and the transverse area of the system. The specific relation assumed to hold between $d E / d y$ and $d N / d y$ only modifies the detailed form of the scaling variable. In other words, the variation of $\gamma_{s}(s)$ with $\epsilon_{0} \tau_{0}$ in Fig. 3 , based on eq. (8), will coincide with that of $\gamma_{s}(s)$ as function of $\left[\left(4 c_{s}^{1 / 3} / 3\right) \epsilon_{0} \tau_{0}\right]^{3 / 4}$, as given by eq. (18). The basic meaning of the observed universal model-independent behavior thus is that $\gamma_{s}$ is fully determined by the ratio between the total transverse energy and the transverse area of the system.

It would evidently be interesting to extend these consideration to hadron production in $e^{+} e^{-}$annihilation. Here the crucial aspect is a determination of the relevant transverse area; work on this is in progress. 
In closing, we recall that our causality considerations relate the size of the causal correlation region to the life-time of the fireball as strongly interacting thermal system. Strangeness suppression provides an experimental measure of the correlation region, while the initial energy density determines the life-time of the interacting thermal medium. The observed scaling of $\gamma_{s}$ with a function of $\epsilon_{0} \tau_{0}$ is thus an observable consequence of our basic causality correspondence.

Nevertheless, if one were to just ad hoc assume a $\gamma_{s}(s)-\epsilon_{0}(s)$ correlation, the results of Figs. 3 and 4 would remain. On a purely phenomenological level, one thus also finds that the degree of strangeness suppression in hadronic and nuclear collisions is fully determined by the initial energy density. A specific example of this (the $K^{-} / \pi$ ratio) was already noted some time ago [20].

\section{Acknowledgement}

It is a pleasure to thank J. Schukraft for some stimulating questions and F. Karsch for helpful comments.

\section{References}

[1] F. Becattini and G. Passaleva, Europ. Phys. J. C23 (2002) 551.

[2] P. Braun-Munzinger, K. Redlich and J. Stachel, in Quark-Gluon Plasma 3, R. C. Hwa and X.-N Wang (Eds.), World Scientific, Singapore 2003.

[3] F. Becattini and R. Fries, arXiv:0907.1031 [nucl-th], and Landolt-Boernstein 1-23.

[4] J. Letessier, J. Rafelski and A. Tounsi, Phys. Rev. C64 (1994) 406.

[5] The $\gamma_{s}$ values were obtained in the following data analyses, where also the original experimental references are given:

F. Becattini, J. Manninen and M. Gazdzicki, Phys. Rev. C 73 (2006) 044905.

F. Becattini et al. Europ. Phys. J. CC66 (2010) 377.

F. Becattini and J. Manninen, Phys. Rev. C78 (2008) 054901 et al. Europ. Phys. J. CC66 (2010) 377.

M. Floris et al., Nucl. Phys. A931 (2014) 103.

F. Becattini, hep-ph/9701275.

F. Becattini et al., Phys. Rev. Lett. 111 (2013) 082302

[6] R. Hagedorn and K. Redlich, Z. Phys. C27 (1985) 541

[7] J. S. Hamieh, K. Redlich and A. Tounsi, Phys. Lett. B 486 (2000) 61.

[8] I. Kraus et al., Phys. Rev. C 76 (2007) 064903.

[9] P. Castorina and H. Satz, Int. J. Mod. Phys. E23 (2014) 4, 1450019.

[10] P. Castorina and H. Satz, arXiv:1601.01454. 
[11] J. D. Bjorken, Lecture Notes in Physics (Springer) 56 (1976) 93.

[12] A. Bazazov et al. (HotQCD Coll.), Phys. Rev. D90 (2014) 094503.

[13] S. Borsanyi et al. (Wuppertal-Budapest Coll.), Phys. Lett. B730 (2014) 99.

[14] J. D. Bjorken, Phys. Rev. D 27 (1983) 140.

[15] K. Aamodt et al. (ALICE Coll.), Phys. Rev. Lett. 105 (2010) 252301.

[16] F. Becattini, J. Manninen and M. Gazdzicki, Phys. Rev. C 73 (2006) 044905.

[17] The $p p$ fit was made using the data given in [5] and shown in Fig. 1.

[18] B. Abelev et al. (ALICE Coll.), Phys. Rev. Lett. 110 (2013) 032301.

[19] C. Blume and C. Markert, Prog. Part. Nucl. Phys. 66 (2011) 834.

[20] Fuqiang Wang, J. Phys. G28 (2001) 2109. 